\title{
Oposición empresarial a las reformas laborales y educativas en México: el caso de Monterrey, 1962-1972
}

\author{
Dr. Óscar Flores Torres \\ Director del Centro de Estudios Históricos \\ El Colegio de Tamaulipas (CEH/EL COLTAM) \\ oflores6o@gmail.com \\ El Colegio de Tamaulipas, México
}

Dra. Magda Yadira Robles magda.robles@udem.edu Universidad de Monterrey, México

Recibido: mayo de 2015

Aprobado: septiembre de 2015

Resumen: En México, el régimen presidencial en México de Lázaro Cárdenas (1934-1940) estableció nuevas reglas de funcionamiento del Estado con respecto a los ciudadanos(as) mexicanos(as). Dos décadas después, los grupos conservadores anti-cardenistas, defendieron abiertamente sus derechos e intereses de forma colectiva. Primero con movimientos de reacción contra la afectación de sus bienes e intereses inmediatos, después para enfrentar la imposición de una ideología que atentaba contra los principios conservadores tradicionales. El epicentro de estos movimientos conservadores (empresarial y religioso católico) se presentó en la ciudad de Monterrey: primero contra el libro de texto gratuito en México en 1962 y posteriormente en 1968 a 1971 contra las demandas de una apertura en la educación superior.

Palabras clave: educación, religión, empresarios, libros de texto y movimientos urbanos.

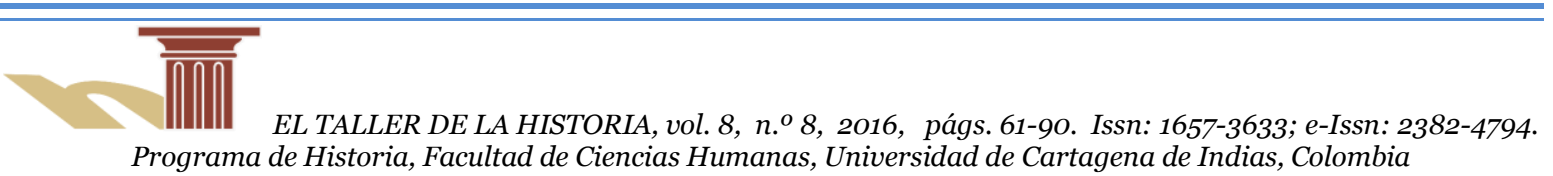


Summary: In Mexico, the presidential regimen of Lazaro Cardenas (1934-1940) established new State functioning rules concerning the mexican citizens. Two decades later, the conservative "anti-cardenistas", openly defended their rights and interests in collective form. First, with reaction movements against the effects of their goods and immediate interests, later to face the imposition of an ideology that threatened against the traditional conservative principles. The epicenter of these conservative movements (business and religious catholic) was presented at the city of Monterrey: first against the free textbook in Mexico in 1962 and later in 1968 to 1971 to oppose the demands of an opening in the college education.

Keywords: education, religion, business, textbooks and urban movements.

\section{Introducción}

En México, después de la etapa armada de la revolución mexicana (1910-1920), se dieron manifestaciones violentas contra el nuevo régimen que intentaba crear una sociedad incluyente. Una de estas etapas fue el régimen presidencial en México de Lázaro Cárdenas (1934-1940) el cual no perjudicó ni pretendió cambiar el sistema económico capitalista del México post-revolucionario, pero si estableció nuevas reglas de funcionamiento. Tres movimientos sociales llaman la atención en este periodo cardenista: el obrero el campesinado y el educativo. Presenciamos la afectación de bienes materiales y de intereses ideológicos a través de las demandas populares o bien de parte de la administración pública, a familias propietarias de tierras, empresarios, a la iglesia católica, entre otros grupos históricamente hegemónicos en México. Tras una aparente calma al final de su mandato, todo parecía que buena parte de la reconciliación social había sido resuelta.

Sin embargo, dos décadas después en 1962, estos mismos grupos conservadores, defendieron nueva y abiertamente sus derechos e intereses de forma colectiva. Primero con movimientos de reacción contra la afectación de sus bienes e intereses inmediatos, después conformando una coalición de agrupaciones empresariales para enfrentar tanto las afectaciones materiales como la imposición de una ideología que atentaba contra los principios conservadores tradicionales. Este fue el caso de los movimientos conservadores empresariales y religiosos en la ciudad de Monterrey contra el libro de texto gratuito en México en 1962; el segundo fue el movimiento 
estudiantil y la guerrilla urbana centrados en los años de 1968 a 1972. El objetivo de esta investigación es analizar el escenario histórico y jurídico de estos conflictos y el impacto que representó para la estabilidad política del país.

\section{La conquista del derecho a la educación en México}

Desde la Constitución de 1857 se estableció la educación elemental obligatoria y gratuita en México. Esta aspiración llegó al posterior texto constitucional de 1917, actualmente vigente. Sin embargo, la gratuidad de la enseñanza venía siendo sólo un término jurídico sin posibilidades reales de cumplimiento hasta mediados de siglo XX, cuando menos en las zonas rurales. Por ello, propusieron en el año de 1959 los libros de texto gratuito con doble objetivo: la difusión del sentimiento nacionalista y una mayor integración de la sociedad mexicana. ${ }^{1}$

Sin duda, la reacción no se hizo esperar. Los cuestionamientos de los grupos opositores urbanos, se centraron en la anticonstitucionalidad de esta medida. Para 1962, el conflicto empezó a rebasar los límites de un problema puramente educativo adquiriendo las dimensiones de un debate nacional. La oposición pasó de la crítica a manifestaciones de protesta con claras implicaciones de carácter sociopolítico. Algunas ciudades del interior del país fueron escenario de estos enfrentamientos. Pero el movimiento urbano contestatario en la ciudad de Monterrey fue el detonador.

\subsection{Primeros proyectos del libro de texto gratuito en México}

Como ya lo comentamos, desde la Constitución Política de los Estados Unidos Mexicanos de 1857, se había establecido la educación elemental obligatoria y gratuita. Esta aspiración había quedado también consignada como precepto legal en la de 1917. Sin embargo, la gratuidad de la enseñanza venía siendo sólo un término jurídico sin posibilidades reales de cumplimiento. Las autoridades educativas estaban

\footnotetext{
${ }^{1}$ Cecilia Graves Laine "Política educativa y libros de texto gratuitos. Una polémica en torno al control por la educación", en Revista Mexicana de Investigación Educativa, mayo-agosto, volumen 6, número 12, Consejo Mexicano de Investigación Educativa, México, 2001, pp. 205-206
} 
conscientes de que mientras los alumnos no pudieran adquirir el material de enseñanza necesario, no podría cumplirse con los principios establecidos por el artículo $3^{\circ}$ de la Constitución.

Ante ello, la idea de distribuir libros de texto oficiales se derivó del proyecto del Secretario de Instrucción Pública, José Vasconcelos a partir de $1921^{2}$. El programa de publicaciones llevado a cabo por el entonces Secretario de Instrucción Pública pretendía hacer llegar las obras de autores clásicos a los más importantes rincones del país. Vasconcelos consideraba que la gratuidad del material de lectura era indispensable porque el pueblo era pobre y no tenía el hábito de la lectura. Posteriormente, a juicio de las autoridades educativas de la década de 1940, los libros escolares eran en muchas ocasiones mediocres y a precios, cada año, más elevados3.

Para contrarrestar precisamente estos abusos se había creado, en 1954, la Comisión Revisora de Libros de Texto y de Consulta (CRLTC) de la Secretaría de Educación, encargada de seleccionar los textos para los seis años de educación primaria y fijar los precios 4 . Sin embargo, cuando se ponían en juego los intereses particulares de autores, editorialistas y libreros, no siempre las obras elegidas eran las adecuadas. A la par, los precios de los mismos iban constantemente al alza afectando a las clases populares y provocando mayores deficiencias en el sistema educativo nacional. Finalmente, hay que comentar, que su tiraje en conjunto solo satisfacía una mínima parte de la demanda escolar.

Tres décadas después, en los años cincuenta, el Secretario de Educación Jaime Torres Bodet, retomó la idea de Vasconcelos y la planteó de forma más ambiciosa. El Estado debía de producir los libros con doble finalidad. En primer lugar, se debía de hacer extensiva la educación a todos los sectores sociales otorgando a todos los alumnos de enseñanza primaria, los medios indispensables para el aprendizaje. $\mathrm{Y}$, en segundo, asegurar una base cultural uniforme para la niñez mexicana. Para lograr ambos propósitos dos eran los conductos: la gratuidad y la obligatoriedad de los textos5.

\footnotetext{
${ }^{2}$ Oscar Flores, Historiadores del México Siglo XX, Selección, presentación y notas de, México, Editorial Trillas, Colección Linterna Mágica, número 33, 2003, pp. 112-113

${ }^{3}$ C. Graves, "Política educativa y libros", p. 206

${ }^{4}$ C. Graves, "Política educativa y libros", p. 206

${ }^{5}$ C. Graves, "Política educativa y libros", p. 206
} 
Para el gobierno federal, sin duda, los libros de texto gratuito eran el mejor conducto para lograr dos principios que el gobierno emanado de la sangrienta revolución iniciada en 1910 -e institucionalizada en 1917-, buscaba ansiosamente: la difusión de un sentimiento nacionalista y a una mayor integración de la sociedad mexicana.

Por otra parte, la cristalización de este proyecto traería en consecuencia un mayor control político sobre el aparato educativo. Fue de esta forma que el presidente Adolfo López Mateos aprobó esta propuesta y, en febrero de 1959, se creó la Comisión Nacional de Libros de Texto Gratuitos (CNLTG). La única observación que hizo el Presidente del país, fue que estos libros no contuvieran "expresiones que susciten rencores, o odios, prejuicios y estériles controversias."6 (sic)

\subsection{El primer libro gratuito en México}

El decreto que creaba esta Comisión tuvo cuidado de no provocar viejas rencillas, particularmente con los grupos conservadores como lo fueron la Iglesia Católica y el empresariado, particularmente el poderoso grupo económico asentado en la ciudad de Monterrey. En otras palabras, en ningún momento, el nuevo proyecto pretendió modificar el contexto educativo vigente sino que mantuvo la neutralidad ideológica de los principios incluidos en la reforma, en 1946, del artículo 30 constitucional. Por otra parte, el documento no hacía alusión alguna al contenido de los nuevos libros sino que la razón fundamental que argumentaba era de carácter económico7.

Así fue como se nombró a Martín Luis Guzmán ${ }^{8}$ como presidente de la CNLTG. Este sería apoyado por un secretario general, seis vocales, doce colaboradores pedagógicos y cinco representantes de la opinión pública designados entre los directores de los principales diarios de la ciudad México. Estos directores representaban a los diarios de mayor tiraje en el país, tales como Excélsior, El Universal, Novedades, La Prensa y de la cadena García Valseca9.

\footnotetext{
${ }^{6}$ C. Graves, "Política educativa y libros", p.p. 207

${ }^{7}$ Ana Cristina Ávila y Virgilio Muñoz, Creación de la Comisión Nacional de Libros de Texto Gratuitos: La perspectiva escolar, 1958-1964, México, Noriega Editores, 1999, p.p. 87, 88

${ }^{8}$ Enrique González Pedrero, Enrique, Los libros de texto gratuito, México, SEP, 1982, p.15

${ }^{9}$ A. Avila y V. muñoz, Creación de la, 1999, p. 88, 89
} 
El siguiente paso consistió en convocar, mediante concurso, a maestros y escritores mexicanos por nacimiento, para participar en la redacción de los libros de primero a sexto grados de educación elemental. Los autores de las obras elegidas serían gratificados con 75 mil pesos. En caso de que los trabajos presentados no reunieran los requisitos necesarios, la Comisión debería designar, bajo contrato, a los autores de los textos. El resultado, después de siete certámenes, los libros no agradaron a la comisión y hubo que contratar a diversos autores para que emprendieran tan magna labor ${ }^{10}$.

Para lograr este objetivo, la CNLTG trabajó intensamente. Debían entregar al iniciarse el curso escolar de 1960, cerca de 16 millones de ejemplares.

El 30 de enero de 1960, la CNLTG publicó en todos los diarios la lista de los libros aprobados para el ciclo que se iniciaba. Esta ocasión solo comprendía los textos correspondientes a quinto y sexto grados, ya que los libros y cuadernos de trabajo para los cuatro primeros años elaborados por la CNLTG, serían repartidos entre febrero y abril del año en curso en todas las escuelas primarias, fueran oficiales o particulares, federales, estatales o municipales; mientras que los restantes, pertenecientes al tercer ciclo de educación primaria estaban en proceso y no podrían entregarse sino hasta el siguiente año escolar ${ }^{11}$.

Paralelamente, la Secretaría de Educación anunció la obligatoriedad del uso del libro de texto gratuito prohibiendo a los maestros exigir a sus alumnos otros que no estuvieran incluidos en las listas oficiales ${ }^{12}$.

\footnotetext{
${ }^{10}$ A. Ávila y V. Muñoz Creación de la, 1999, p.p. 90

${ }^{11}$ Los alumnos de primero y segundo grados recibirían un libro de lectura y un cuaderno de trabajo que comprendía conocimientos básicos sobre lengua nacional, aritmética, historia, civismo, geografía y ciencias naturales. A partir de $3^{\circ}$, recibían cuatro textos con sus respectivos trabajos. C. Graves, "Política educativa y libros", p.p. 207, 208

${ }^{12}$ C. Graves, "Política educativa y libros", p.p. 208
} 


\subsection{Empresarios e Iglesia: repudio al libro de texto gratuito (1962)}

$\mathrm{Al}$ conocerse la noticia, la reacción no se hizo esperar. Surgieron grandes movilizaciones que involucraron a grupos por demás heterogéneos. En buena medida esta agitación reflejó la antigua rivalidad entre el Estado y la Iglesia católica por el control del sistema educativo.

La lucha surgió primero en el terreno de la legalidad por los directamente afectados por la medida estatal. Nos referimos específicamente a los autores de los textos escolares y a los libreros y editores.

En efecto, la crítica y los cuestionamientos se centraron en la inconstitucionalidad de esta medida. Ante esta reacción, la Secretaría de Educación reiteró el carácter obligatorio de los textos y dio a conocer, a través de un comunicado de prensa, las sanciones penales y administrativas para quienes boicotearan el proyecto de la CNLTG.

Los maestros que trataran de impedir el uso de los libros de texto gratuitos editados por la SEP se exponían a cárcel, multa, destitución e inhabilitación para desempeñar puestos federales. Las escuelas particulares tampoco escapaban a estas sanciones; se les recordaba que de acuerdo con la Ley Reglamentaria del artículo $3^{\circ}$, la SEP podía retirarles la concesión que se les había otorgado. Pese a las amenazas, las denuncias continuaron. Nuevos actores se unieron al debate. La Unión Nacional de Padres de Familia (UNPF), organización fundada en 1917 de marcado corte tradicionalista, dio a conocer en un comunicado de prensa, su inconformidad por las medidas tomadas por la SEP..$^{13}$

El Partido Acción Nacional (PAN), de filosofía conservadora y de marcado tinte católico, se sumó también a la ofensiva. Si bien en un primer momento su crítica fue moderada, al subir el tono de las denuncias de otros grupos sociales, el PAN cambió su discurso en torno al autoritarismo y antidemocracia del proyecto de los libros de texto gratuitos.

Es cierto, que tras la experiencia de la educación socialista, los grupos conservadores habían mantenido una fuerte desconfianza hacia la política educativa del Estado. La reforma al artículo $3^{\circ}$, aprobada en 1946 -la cual eliminaba el concepto "socialista" de la educación-, no había calmado del todo sus temores a pesar de que

\footnotetext{
${ }^{13}$ C. Graves, "Política educativa y libros", p. 214, 215
} 
un régimen de tolerancia privaba en las escuelas particulares. De hecho se llegó a orquestar una intensa campaña anticomunista que se llevó a cabo a través de los principales diarios del país, exigiendo el respeto a la libertad de enseñanza ${ }^{14}$.

Para 1962, el conflicto empezó a rebasar los límites de un problema puramente educativo adquiriendo las dimensiones de un debate nacional. La oposición pasó de la crítica del autoritarismo del Estado a realizar masivas manifestaciones de protesta con claras implicaciones de carácter sociopolítico. Algunas ciudades de México como Puebla, Guadalajara y Morelia fueron escenario de los enfrentamientos entre grupos antagónicos dándose el caso, en algunas escuelas, de confiscar los libros y quemarlos. Pero sin duda alguna los grupos conservadores asentados en la ciudad norteña de Monterrey fueron los detonadores.

\subsection{Los empresarios de Monterrey se manifiestan}

En efecto, el 2 de febrero de 1962, la agrupación tradicional local denominada "Unión Neolonesa de Padres de Familia" convocó a una manifestación para protestar en contra de la imposición de los textos gratuitos ${ }^{15}$. A este llamado acudieron grupos por demás heterogéneos: miembros de organizaciones católicas, del PAN, de la banca y la industria, trabajadores y obreros, todos ellos bajo la consigna "iMéxico sí!" "iComunismo no!". La magnitud de esta concentración, que congregó alrededor de cien mil personas, -la ciudad tenía una población de 601,100 habitantes'16- obligó que las autoridades educativas abandonaran su postura indiferente. Prevalecía el temor de que este tipo de protestas se extendiera hacia otras regiones del país ${ }^{17}$.

Ante esta situación, las manifestaciones se habían vuelto una seria amenaza para la estabilidad política del país. ${ }^{18}$ Por ello, los funcionarios de la SEP aceptaron dialogar. En las negociaciones entre los representantes de los padres de familia y las

\footnotetext{
${ }^{14}$ Hemeroteca de El Norte [HEN], El Norte, Monterrey, primera sección, marzo-diciembre de 1946, p.p. 1 y 2

${ }^{15}$ HEN, El Norte, Monterrey, 2 de febrero de 1962, p.1

${ }^{16}$ Dirección General de Estadística, Censos Generales de Población, 1960, 1970, 1980 y 1990.

${ }^{17}$ HEN, El Norte, Monterrey, 3 de febrero de 1962, p.1

18 David L. Raby, "La "Educación socialista en México", en Cuadernos Políticos, número 29, México, D. F., Editorial Era, julio-septiembre, 1981, pp. 77-78.
} 
autoridades quedó de manifiesto que la oposición al texto obligatorio no era por su contenido mismo, ya que quienes lanzaban los ataques mostraron su desconocimiento absoluto, sino por la naturaleza de la acción del Estado. Con este pretexto, las conversaciones fueron suspendidas ${ }^{19}$. Sin embargo, contrariamente a lo que se hubiere esperado, el conflicto no se agudizó.

La actitud conciliadora por parte de las autoridades educativas quedó de manifiesto cuando declararon públicamente que la obligatoriedad de los libros no implicaba únicos o exclusivos, ya que quienes tuvieran las posibilidades podían comprar libros de texto complementarios para sus hijos, siempre y cuando fueran autorizados por la SEP20.

A partir de entonces, los ánimos se tranquilizaron. El interés por continuar el debate fue disminuyendo paulatinamente. En cierta forma los grupos opositores a los textos únicos habían logrado sus objetivos, aunque sólo parcialmente. Con el paso del tiempo, la obligatoriedad de los libros no volvió a ser cuestionada incluso cuando, en 1973, se decidió la elaboración de una nueva versión de los textos gratuitos.

Al finalizar el sexenio del presidente Adolfo López Mateos (1964), la Comisión había logrado editar y distribuir más de 112 millones de ejemplares de libros de texto y cuadernos de trabajo ${ }^{21}$. Con esta medida, el Estado cumplía cabalmente con el precepto de gratuidad establecido en el artículo $3^{\circ}$ Constitucional pero, al mismo tiempo, mantenía su hegemonía en el ámbito educativo. Más allá de este conflicto que representó un gran desafío a la estabilidad política del país, es innegable el acierto de la distribución de los textos gratuitos a todos los niños mexicanos; para muchos han sido y siguen siendo, el único recurso para su aprendizaje.

\section{Movimientos urbanos 1968-1972}

En este período destacan en la ciudad de Monterrey los movimientos estudiantiles de educación media y superior -que posteriormente alimentarían la guerrilla urbana, así como los movimientos populares de los "sin tierra".

\footnotetext{
${ }^{19}$ C. Graves, "Política educativa y libros", p. 217.

${ }^{20}$ David L. Raby, "La "Educación socialista", p.79.

${ }^{21}$ C. Graves, "Política educativa y libros", p. 218
} 


\subsection{El movimiento universitario}

El movimiento universitario iniciado en México en 1965-1966, que desembocó en la represión del 2 de octubre de 1968 en la Plaza de las Tres Culturas en la ciudad de México, se ha ido enriqueciendo en el imaginario popular como un lugar para dar guardar sueños, aspiraciones, logros y frustraciones de toda una generación ${ }^{22}$. Este lugar común se convirtió en un modelo de lucha. A tal grado, que los movimientos estudiantiles posteriores en la UNAM, particularmente el del Consejo Estudiantil Universitario (CEU) y el Consejo General de Huelga (CGH) de los años de 1986 y 1999-2000 respectivamente, han querido emularlo casi al detalle, pero sin éxito.

Este conflicto de 1968, que indudablemente impactó en la conciencia popular del México contemporáneo, no se trató de un conflicto al que haya precedido una crisis económica, por ello las explicaciones sociales contextuales internas y externas a la Educación Media y Superior son las que han predominado desde los años setenta. Sin embargo, también existen interpretaciones que apuntan a razones políticas $^{23}$. Estos conflictos no tardarían en aparecer. El disentimiento a esta política estaba sumamente localizado en el noreste de México. Para 1969, la élite política, empresarial y sus organismos corporativos en Monterrey, mostrarían al presidente Echeverría como se manejaban las cosas en esta ciudad de las "industrias de fuego."

\footnotetext{
${ }^{22}$ Véase sobre este tema entre otros a: Olivares Ortega, Octubre dos: historias del movimiento estudiantil, México, UAM-Unidad Xochimilco, 1988; Héctor Aguilar, Subversiones silenciosas: ensayos de historia y política de México, México, Aguilar, 1993; Héctor Aguilar, La guerra de Galio, México, Cal y Arena, 1992; Héctor Aguilar, Después del milagro, México, Cal y Arena, 1989; Gilberto Guevara, La democracia en la calle, México, Siglo XXI Editores, 1986; Gilberto Guevara, "El 68 y la Universidad de México", en Revista de la Universidad de México, XXXIII, diciembre de 1978enero de 1979, pp. 4 y 5; Sergio, Zermeño, México: una democracia utópica. El movimiento estudiantil del 68, México, Siglo XXI, 1978; Luis González de Alba, Luis, Los días y los años, México, Era-SEP, 1971 y Ramón Ramírez, El movimiento estudiantil de México, julio-diciembre de 1968, 2 vols. México, Era, 1969.

${ }^{23}$ Javier González Rubio (coordinador), México, 30 años de movimiento, México Universidad Iberoamericana, 1998.
} 


\subsection{El movimiento universitario en Nuevo León}

Lo sucedido en Monterrey entre 1968 y 1972, en cuanto al movimiento universitario se refiere, no se puede explicar sin el conocimiento de este contexto nacional. Fue ésta ciudad la protagonista nacional de esta tensión social que marcó la transición de un régimen autoritario, como el del presidente Gustavo Díaz Ordaz (1964-1970), a uno aperturista como el del presidente Luis Echeverría Álvares, más no tolerante al pensamiento conservador de los grupos económicos y políticos de esta región.

No hay duda que el proyecto de transición del grupo político a nivel nacional fue puesto a prueba desde sus inicios en Monterrey. Prueba de esto, el gobernador Eduardo A. Elizondo (1967-1971) -apoyado por la extensa y poderosa oligarquía empresarial que controlaba la industria regiomontana-, planteó modelos alternos para enfrentar la problemática de la Educación Superior no solo en Monterrey sino en el ámbito nacional.

El modelo de una nueva universidad pública con características particulares de las universidades privadas, fue lo que llegó a plantear Elizondo desde 1968, adelantándose a las demandas (aunque con otro tenor) del movimiento estudiantil nacional. Este proyecto de largo alcance (lo volvería a plantear en 1969 con la autonomía y en 1971 con una nueva Ley Orgánica para la UANL), ha sido relegado y minimizado por los investigadores del movimiento universitario en México, cuando han llegado siquiera a mencionarlo. Considerando que la tendencia fue a magnificar los logros y fracasos del pensamiento y acciones de la izquierda política en éste período, el pensamiento conservador y alternativo en este país, ahora de moda, fue menospreciado y vituperado 24 .

Las reformas por las que atravesaron universidades públicas en todo el país a partir de la década de 1990, no disienten en casi nada a lo esbozado por Elizondo y el grupo político y empresarial de Nuevo León en 1968. Entre sus planteamientos están los siguientes: la necesidad de un financiamiento directo a las Universidades por medio de los aumentos de cuotas y de becas préstamos a fin de ser pagados sin intereses al final del período de estudio, siempre y cuando el alumno muestre su ingreso al mundo laboral; el examen de admisión como forma de seleccionar a los alumnos que realmente tienen potencial de estudiar y aprovechar de mejor manera

\footnotetext{
${ }^{24}$ Sergio Zermeño, México: una democracia utópica. El movimiento estudiantil del 68, pp. 256-283
} 
los recursos universitarios; líneas y proyectos de investigación financiados con alta productividad; el estímulo a los proyectos universitarios que tienen un éxito comprobado en el sector productivo y social; y la elevación del nivel académico de sus profesores a través de un sistema de evaluación de su productividad en investigación, desarrollo de recursos humanos, grados académicos obtenidos, así como de una retroalimentación del propio alumno al que imparte cátedra.

Otra de las reformas estimuladas por Elizondo a fin de enfrentar la crisis de la Educación Media y Superior que tuvo México en 1968, fue el llamado a la creación de universidades privadas que mostraron su capacidad de competitividad en el mundo académico, incluso hasta la actualidad. Un ejemplo de ello son: la Universidad de Monterrey y la Universidad Regiomontana, creadas ambas en 1969.

Sin embargo, parte del proyecto fue truncado debido a la oposición interna organizada de la propia población universitaria (más no mayoritaria, ya que ésta "población silenciosa" se mantuvo al margen de toda participación); por los grupos locales ligados al Partido Revolucionario Institucional (PRI) de obreros y campesinos y; por las autoridades federales a partir de 1971.

Este modelo alterno de Universidad pública, que fue visto como la pérdida tanto de la autonomía como de la verdadera vocación universitaria ${ }^{25}$, retrasó por casi 25 años el inicio de las actuales reformas universitarias.

Es tiempo de hacer una nueva revisión de estos acontecimientos y apreciar sin arrebatos extremos lo sucedido entre 1968 y 1972 en nuestro entorno universitario. Las pasiones relegaron proyectos y esperanzas de mejora y crecimiento académico, solo por el hecho de encasillarlos en ideologías no compatibles con la efervescencia política izquierdista que invadió la Universidad de Nuevo León a partir de 1969.

\footnotetext{
${ }^{25}$ Oscar Flores, La Autonomía Universitaria, 1968-1971, Monterrey, UANL, 2012, pp.116, 117 y 118.
} 


\subsection{La creación de nuevas universidades privadas}

El gobierno de Nuevo León, la potente elite empresarial y los diversos grupos religiosos organizados de la ciudad, contemplaron desde fines de 1967 la crisis universitaria por venir. La Educación Superior en México estaba por experimentar un boom y había que estar preparado para presentar nuevas alternativas más allá de las instituciones establecidas como eran la Universidad de Nuevo León (UNL) y el Instituto Tecnológico y de Estudios Superiores de Monterrey (ITESM). El gobernador del Estado abrió esta posibilidad de forma pública el 18 de enero de 1968. En esa ocasión dijo que todos los planteles educativos particulares que desearan participar en la Enseñanza Superior, encontrarían altos estímulos de parte de su administración. ${ }^{26}$ El llamamiento fue dado después de que dos grupos particulares mostraron interés en fundar instituciones de Educación Superior en la ciudad. Esta iniciativa daría lugar a la fundación en 1969 de la Universidad de Monterrey (UDEM) y la Universidad Regiomontana (UR). Un año después se crearía el Centro de Estudios Universitarios (CEU).

Para 1972, estas tres Universidades más el ITESM fundado en 1943, tenían 21,139 alumnos inscritos en sus carreras profesionales, ${ }^{27}$ en contraposición a los 22,000 alumnos que tenía la UANL en sus instalaciones de Educación Superior ${ }^{28}$. En otras palabras, las Instituciones de Educación Superior privadas tenían el 49 por ciento del alumnado total inscrito en el Estado, mientras la UANL poseía el restante 51 por ciento. En contraposición a la situación existente en el resto del país, el cual presentaba una composición del 10 por ciento y 90 por ciento, respectivamente. ${ }^{29}$

En este sentido, el proyecto del gobernador Elizondo tuvo un éxito inmediato con la apertura de la Educación Superior al el capital privado. Lo que demuestra que el sector empresarial y comercial de Monterrey estaba sumamente interesado en el

\footnotetext{
${ }^{26}$ HEN, El Norte, Monterrey, 19 de enero de 1968, p.1

${ }^{27}$ Para noviembre de 1972, la distribución de alumnos en las Instituciones de Educación Superior privadas en Monterrey estaba como sigue: el ITESM tenía 12,250 alumnos; el CEU, 3,487; la UDEM, 3,402 y la UR 2,000 alumnos. HEN, El Norte, Monterrey, 30 de noviembre de 1972, p.1. Oscar Flores, Universidad de Monterrey. Historia y Desafíos (1969-2004), Editorial Trillas, México, 2004, p. 200

${ }^{28}$ O. Flores, Universidad de Monterrey, p.p. 200, 2001.

${ }^{29}$ Susana García y Liliana Vanella (1979), Política educativa y valores nacionales, México, Nueva Imagen, 1979, p.p. 36-39.
} 
financiamiento de la Educación Superior, a diferencia de lo que suponen los investigadores sobre este tema ${ }^{30}$.

\subsection{La lucha por la Autonomía}

Un año después de que la propuesta de autofinanciamiento de la UNL fue rechazada se inicia a mediados del año de 1969 un movimiento estudiantil, magisterial y sindical que desembocó en la necesidad de otorgar la Autonomía a la Universidad de Nuevo León ${ }^{31}$. Tras numerosas presiones y ante la generalización del conflicto universitario, el Gobierno del Estado fue receptivo a las peticiones del movimiento. El Ejecutivo envía el 22 de octubre al H. Congreso del Estado una iniciativa a fin de reformar los artículos 16 y 24 de la Ley Orgánica de la UNL; la reforma fue encaminada a que el rector fuera designado por una asamblea de profesores y alumnos y para que directores de Escuelas y Facultades fueran nombrados en las Juntas Directivas. La noticia de esta Autonomía de la Universidad, fue dada a conocer por el gobernador Elizondo ante un grupo de estudiantes universitarios. Ese día decide la destitución del rector, del secretario general y los 24 directores de la ahora UANL. Para el 23 de octubre, la destitución masiva de las autoridades universitarias es un hecho. Algunos profesores decanos se hacen cargo de las Facultades, pero el resto no aceptan renunciar por considerar que la reforma a la Ley Orgánica, sólo es una iniciativa. La propuesta del gobernador crea un divisionismo interno de parte del profesorado. Un grupo de profesores encabezados por el doctor Agustín Basave Fernández del Valle, se afilia en una organización denominada Asociación de Maestros de la UNL (compuesta por 200 profesores) que desconoce la iniciativa. ${ }^{2} \mathrm{El} 24$ de octubre acuden ante el Congreso y le piden no aprobar la iniciativa del Gobernador. Consideran que tales modificaciones pueden traer consecuencias graves a la estabilidad de la UNL. Ese mismo día, el Consejo Estudiantil toma rectoría a fin de presionar a

\footnotetext{
${ }^{30}$ Véase Pablo Latapí, Política educativa y valores nacionales, Editorial Nueva Imagen, México, 1979, p.p. 85-108.

${ }^{31}$ O. Flores, La Autonomía Universitaria, p.p. 133-152.

${ }^{32}$ Véase el comunicado de la creación de esta asociación, dirigida a los universitarios en HEN, El Norte, Monterrey, 24 de octubre de 1969, p.3
} 
las autoridades estatales para que aprueben la iniciativa. Se une al Consejo Estudiantil el Sindicato de Trabajadores de la UNL, los cuales acuden el 25 de octubre al Congreso a pedir la aprobación de las reformas y acusar de elementos disolventes a la nueva asociación de profesores. La autonomía se vuelve la bandera del movimiento estudiantil y de los trabajadores y empleados de la UNL.

Los estudiantes exploran creativos y novedosos métodos de lucha y protesta. $\mathrm{El} 4$ de noviembre estos desfilaron por la noche con antorchas, mientras acusaban al Congreso del Estado de tratar de impedir con su tardanza, la reforma universitaria. Al día siguiente, el Consejo Estudiantil sesionó para definir los métodos de lucha. Ese día se pintan camiones y se reparten volantes de sus demandas a la población citadina. El presidente del Congreso lamenta que el movimiento intente manipular al poder legislativo y exige a los estudiantes el regreso a clases como prerrequisito para estudiar y dictaminar la iniciativa del gobernador. 33 La tardanza resolutiva del Congreso -el cual evidencia divisiones internas entre sus ocho integrantes-, favorece la llegada de nuevas iniciativas por parte de grupos políticos dentro y fuera de la UNL. El STUNL, también manifiesta que estudia las reformas a la Ley a fin de presentarlas a los legisladores, y cuestiona la elaboración de las Leyes Orgánicas por grupos políticos que se adjudican "el derecho de representación universitaria para la elaboración de la mencionada Ley." 34 No hay duda, para principios de noviembre de 1969, los universitarios pusieron contra la pared al poderoso Gobierno Estatal.

Finalmente ante la presión estudiantil, de la población y del Ejecutivo (el cual agiliza la cesión de la Autonomía al conocer ese día el anuncio del destape del candidato a la presidencia de la república, Luis Echeverría), el Congreso del Estado reforma el 10 de noviembre de 1969, los artículos 16 y 24 de la Ley Orgánica de la UNL, pero no cede la paridad de votos que propuso Elizondo en su iniciativa original. La designación de Luis Echeverría Álvarez como candidato del PRI a la presidencia, el mismo día que se resuelve dar la Autonomía a la Universidad a través de la reforma a los artículos 16 y 24 de la Ley Orgánica, no es del todo gratuito. La relación de tensión que se vivía en la entidad por la cuestión universitaria, no debía alargarse demasiado, en vista de la designación de un candidato presidencial favorable al respeto de la autonomía, a fin de desligarse de la tutela del presidente en funciones, Gustavo Díaz Ordaz. Así lo visualizó Elizondo y es muy probable que haya presionado

\footnotetext{
${ }^{33}$ El Norte, Monterrey, 5 de noviembre de 1969, p.1

${ }^{34}$ Véase "Sobre el Proyecto de Reforma a los Artículos 16 y 24 de la Ley Orgánica", HEN, El Norte, Monterrey, 10 de noviembre de 1969, 1-B
} 
al Congreso a fin de acelerar la "desincorporación" de la Universidad y, por lo tanto, de sus problemas internos, del Gobierno del Estado.

Los legisladores propusieron que el Ejecutivo del Estado nombrara un rector provisional en tanto el Consejo de la UNL designara el definitivo.35 El Gobernador comentó que promulgaría y publicaría la Ley, en cuanto la Universidad volviera a la vida normal, para lo que pidió como única medida simbólica la entrega de la Torre de Rectoría por parte de los estudiantes -quienes la tenían ocupada- , a fin de que el rector provisional pudiera asumir su cargo. 36

Después de meses de lucha de parte de los universitarios en busca sistemática de la autonomía las reformas a los mencionados artículos quedaron estipulados en el Periódico Oficial.37 Sin embargo, la reacción universitaria ante la mutilación de las reformas por parte de los legisladores, es de reproche. El Consejo Estudiantil 38 y el STUNL rechazaron las reformas (estos aclaran que la paridad de votos era para las funciones eleccionarias más no académicas). En último momento, Elizondo envió una nueva iniciativa de reforma a la Ley (la cual la expone personalmente ante el Congreso en pleno) para satisfacer a los universitarios. El 18 de noviembre, el Congreso del Estado aprueba con dispensa de trámite, la iniciativa del gobernador para crear una Comisión con representación paritaria de maestros y alumnos que redacte el Proyecto de una nueva Ley Orgánica de la UNL, en un plazo que vencería el 31 de mayo de 1970. La iniciativa aprobada también incluía una modificación al artículo 31 de la Ley Orgánica de la UNL para que se crearan las Juntas Directivas de cada Facultad o Escuela en forma semejante al Consejo Universitario, y en las que los estudiantes representaran la tercera parte de los votos. 39 Esta última modificación no fue la original enviada por Elizondo, ya que éste si especificó la paridad en 50 por ciento 40 .

\footnotetext{
${ }^{35}$ Véase Hemeroteca Periódico El Porvenir (HEP), El Porvenir, Monterrey, 11 de noviembre y HEN, El Norte, Monterrey, 11 de noviembre de 1969

${ }^{36}$ HEP, El Porvenir, Monterrey, 11 de noviembre y HEN, El Norte, Monterrey, 11 de noviembre de 1969

${ }^{37}$ Archivo General del Estado de Nuevo León (AGENL), Periódico Oficial del Estado de Nuevo León, Monterrey, 11 de noviembre de 1969

${ }^{38}$ Véase HEN, El Norte, Monterrey, 11 de noviembre de 1969

${ }^{39}$ AGN, Periódico Oficial del Estado de Nuevo León, Monterrey, 26 de noviembre de 1969

${ }^{40} \mathrm{El}$ artículo transitorio único que presentó Elizondo personalmente ante el Congreso exponía lo siguiente: "Conforme a las disposiciones de la Ley Orgánica de la UNL mientras se elijan los nuevos
} 
Finalmente, tras un largo proceso de movilización y trabajo en cada una de las Facultades y Escuelas, representantes de la izquierda universitaria obtuvieron el consenso para dirigir los órganos de gobierno de la UANL.

Para 1970, Elizondo consideraba que el Estado ya no debía de financiar de manera creciente a la Universidad, y así lo manifestó al secretario general del STUANL, Carlos Ruiz Cabrera, cuando lo visitó a principios de ese año a fin de explicar la importancia de las peticiones del sindicato. Ante un gobernador adusto, Cabrera escuchó en voz de Elizondo por primera vez, una negativa rotunda. El gobierno - para Elizondo- había dado los pasos necesarios para crear la autonomía, había estimulado la organización universitaria a fin de modificar y discutir una nueva Ley Orgánica, y había aumentado el subsidio de manera creciente durante su mandato, pero el asunto había llegado a su límite: correspondía a los universitarios a buscar nuevas formas de financiamiento o bien comprender que la Universidad ya no podía seguir creciendo. Acusó a Cabrera y a los universitarios inconformes de creer que tenían ante sí una empresa comercial o industrial, que esto no solo no era así, sino que además no les pertenecía, sino que debían su trabajo y su labor a la comunidad nuevoleonesa, la cual daba sustento a tal empresa educativa.

La Educación superior era importante, pero el gobierno estatal tenía otras prioridades educativas en la educación básica. De los 284 millones de pesos destinados en 1970 a la Educación en el Estado (estos representaban el 62.88 por ciento del presupuesto total del Estado), solo 66 correspondían a la UANL, el resto se destinaba a los otros niveles educativos. ${ }^{41}$

Por su parte, el proyecto político de la izquierda universitaria triunfante en la UANL, recibiría el bautismo del régimen el 10 de junio de 1971. En efecto, nos referimos al conocido "Jueves de Corpus".

titulares de la autoridad universitaria, los maestros decanos de cada Facultad o Escuela quedarán al cargo de las respectivas Direcciones. Por lo que hace al Rector, los mencionados maestros decanos y UN NÚMERO IGUAL DE REPRESENTANTES ESTUDIANTILES seleccionarán a un rector provisional, a reserva de que el definitivo sea nombrado". Véase HEN, El Norte, Monterrey, 19 de noviembre de 1969

${ }^{41}$ HEN, El Norte, Monterrey, 21 de mayo de 1970, 1-B 


\subsection{La represión del Jueves de Corpus}

Para muchos historiadores y politólogos, la represión de la manifestación estudiantil organizada por el Instituto Politécnico Nacional en apoyo al movimiento de Nuevo León, el 10 de junio de 1971, fue una consecuencia de lo sucedido a Elizondo y al grupo político y económico de Monterrey42. Sin embargo, ¿̇cómo pudo haber sido una consecuencia, si precisamente este contingente estudiantil se trasladó a la ciudad de México a fin de difundir y mostrar al desalentado movimiento estudiantil su éxito contra el embate conservador en Monterrey y el apoyo recibido del centro?

El regente del Departamento del Distrito Federal (DDF), Alfonso Martínez Domínguez, siempre negó la existencia del grupo Halcones43. Sin embargo, el efecto político de este acontecimiento, terminó con el despido del Regente del DDF, Alfonso Martínez Domínguez, de Julio Sánchez Vargas, procurador general de la república y Rogelio Flores Curiel, jefe de la policía capitalina en junio de 1971, entre otros. 44

La explicación radica en la política practicada hacia los movimientos de izquierda por parte del régimen de Echeverría. En efecto, se intentó encauzar al movimiento estudiantil post68 hacia peticiones y demandas que el propio gobierno pudiera cumplir, pero a cambio de una alianza estrecha entre su gobierno y estos grupos, a fin de poder enfrentar los conflictos con los empresarios nacionales. La alianza se dio al principio del régimen entre el gobierno federal y los grupos de izquierda colaboracionistas, a fin de detener el proyecto de Elizondo en Nuevo León. Pero una vez que se dio la renuncia de Elizondo y se debilitó momentáneamente este proyecto, el régimen federal no estaba dispuesto a enfrentar demandas y consignas más allá

\footnotetext{
${ }^{42}$ Alfonso Martínez Domínguez dio una conferencia de prensa el 10 de junio de 1971 a partir de las 21:00 horas y negó la existencia de este grupo paramilitar. Este grupo le fue heredado del anterior regente de nombre Alfonso Corona del Rosal

${ }^{43}$ Sergio Aguayo, La Charola. Una historia de los servicios de inteligencia en México, México, Grijalbo, 2001, p.p. 75-79. Este autor calcula que son 80 millones de tarjetas con documentación de 4 millones de actores políticos, además de 26 mil videos y 250 mil fotografías. Sergio Aguayo, Zamora, 31 de julio de 2002, Entrevista

${ }^{44}$ Oscar Flores, (2008), Del movimiento universitario a la guerrilla, en Verónica Oikón y Martha Eugenia García, Vol I, El Colegio de Michoacán/CIESAS, Zamora, p.p. 486-488, p.p. 473-475
} 
de lo que podía ofrecer la apertura política de 1970 a 1976. En estos casos, la represión fue utilizada indistintamente hacia los movimientos que pretendían cuestionar la actividad política del régimen.

A pesar de esta represión violenta y sangrienta, el rector de la UANL, Héctor Ulises Leal (rector entre los años 1971-1972) nunca se atrevió a cuestionar de forma contundente la política seguida por el gobierno de Echeverría, incluso más, cada vez que requirió apoyo del centro para enfrentar su creciente oposición interna, viajó a México a fin de refrendar su alianza con la política emanada en "Los Pinos".

La represión del jueves de Corpus, selló definitivamente la confrontación entre el gobierno y una parte de los jóvenes estudiantes de educación media y superior en las Universidades, Tecnológicos y Normales rurales del sistema de Educación Pública y Privada, así como de los movimientos campesinos a lo largo y ancho del país. A juicio de un exmilitante de la "Liga Comunista 23 de Septiembre", "se fortaleció entre estos sectores, la idea de que no había otra salida para resolver los conflictos que la autodefensa armada".45 Este fue el caso de la guerrilla urbana en Monterrey.

\subsection{Del movimiento universitario a la guerrilla, 1968-1973}

Estas acciones guerrilleras eran nuevas en el México de segunda mitad de siglo. A partir del asalto al cuartel del Ejército Mexicano en Madera, Chihuahua, el 23 de septiembre de 1965, por la organización llamada Grupo Popular Guerrillero (GPG) encabezada por Arturo Gámiz, los grupos guerrilleros proliferaron ${ }^{46}$.

En el caso de Nuevo León, los acontecimientos estudiantiles locales alimentaron la creación de varios grupos radicales de izquierda que pasaron a la lucha armada. Entre otros están: las Fuerzas de Liberación Nacional (FLN), los Procesos, el

\footnotetext{
${ }^{45}$ M. Ramírez, "La relación de la Liga Comunista 23 de septiembre y el Partido de los Pobres en el Estado de Guerrero en la década de los 70's", en el Foro de Discusión Académica La guerrilla en las regiones de México, siglo XX, Zamora, El Colegio de Michoacán, 28 de julio de 2002, p. 3.

${ }^{46}$ José Luis Sierra (2002), "Fuerzas armadas y contrainsurgencia (1965-1982)", p.p. 23-24. en Foro de Discusión Académica, y Minerva Armendáriz, (2001), Morir de sed junto a la fuente, México, Edición de la autora (Testimonio), 2001, p.p. 71-72
} 
Movimiento Espartaquista, la Liga de Comunistas Armados (LCA) y por supuesto a la Liga Comunista 23 de Septiembre.47

La Liga Comunista 23 de septiembre, fue la organización más compleja y sólida de su época. Este grupo, tomó el nombre compuesto por la fecha del asalto al cuartel Madera a sugerencia de Salas Obregón y también de la Liga de los Comunistas creada por Carlos Marx y Federico Engels en el año de 1848. Este grupo sumamente complejo, se estructuró como un ejército guerrillero. Se creó una Coordinadora Nacional, la cual la integraron dirigentes de las diversas agrupaciones políticomilitares fundadoras. El Buró político lo encabezó Ignacio Salas Obregón, quién tomó la dirección de la organización, junto con José Ángel García Hernández, Rodolfo Gómez García y Manuel Gómez García. El brazo militar lo dirigía el Comité Militar, dividido en dos esferas, una de Comandos y otra de Sanidad. Con influencia nacional, sobre la base de Comités Regionales y Locales, alcanzó la estructura más estructurada (tal vez con excepción del Frente de Liberación Nacional) del movimiento guerrillero de la época. Con su dirigente, Salas Obregón, aprehendido en un enfrentamiento armado el 24 de abril de 1974 y virtualmente declarado desaparecido por las fuerzas de seguridad nacional en mayo de 1974, la Coordinación Nacional se desarticuló. El Consejo de Redacción del periódico Madera, tomó finalmente la dirección de la Liga, la cual sobrevivió (cuando menos su brazo armado) hasta 1982.48

\subsection{Las actividades guerrilleras en Nuevo León}

Entre los meses de septiembre y octubre de 1971, se mostró la otra faceta del movimiento universitario en Monterrey: la guerrilla urbana. El Grupo Procesos tomó la decisión de actuar de manera organizada y bajo una rígida estructura militar en una serie de asaltos bancarios: fueron asaltados el Banco Regional de Norte, una camio-

\footnotetext{
${ }^{47}$ O. Flores, "Del movimiento universitario", en Verónica Oikión y Marta Eugenia García, coordinadoras, Movimientos armados, p.p. 475-479.

${ }^{48}$ Entre 1978 y 1982, 244 guerrilleros se acogieron a la Ley de Amnistía y recibieron su libertad. Hugo Esteve Díaz, Las armas de la utopía. La tercera ola de los movimientos guerrilleros en México, México, Instituto de Proposiciones Estratégicas, 1996, p. 72
} 
neta bancaria del Banco de Nuevo León, y dos supermercados: "Lozano" y "Azcúnaga" en Monterrey49. Convencidos de no haber sido descubiertos en estos atracos, los Procesos dirigidos por Raúl Ramos Zavala decidieron realizar un triple asalto bancario en Monterrey en enero de 1972. Dos comandos lograron entrar con éxito a la acción, un tercero, debido a problemas de coordinación recibió la orden en el último minuto de no ejecutar el tercer asalto.50 Esos dos fueron realizados el viernes 14 de enero, fueron asaltados dos bancos en la ciudad, una sucursal de BANAMEX y el Banco Comercial. El doble asalto bancario cuyo botín fue de 67 mil pesos, fue realizado por un par de comandos guerrilleros conformado por universitarios que habían estado participando desde los años de 1969-1971 en el movimiento de reforma universitaria; ahora, habían mostrado abiertamente su decisión de seguir por la vía de las armas. ${ }^{1}$

Un comando era el autodenominado Pablo Alvarado, el cual lo integraban con nombres ficticios Víctor (cuyo nombre real era Héctor Escamilla), David (Raúl Ramos Zavala), Nacho (Ricardo Morales Pinal), Poncho (Alberto Sánchez) y Vicente (Pedro Morón Chiclayo). Por otra parte se encontraba el comando denominado Carlos Lamarca integrado por Pablo (Gustavo Hirales52), Javier (José Luis Rhi Sausi), Pedro (Jorge E. Ruiz Díaz), Jorge (Luis Ángel Garza) y Jaime (Rodolfo Rivera Gamiz). 53

A raíz de las pistas recabadas después del doble asalto del 14 de enero, la policía judicial localizó a estos comandos en distintas casas de seguridad del área metropolitana de la ciudad de Monterrey. El lunes 17 de enero tras un operativo envolvente y coordinado por la policía judicial se detuvieron numerosos estudiantes y se

${ }^{49}$ Fernández Menéndez, Jorge, Nadie supo nada. La verdadera historia del asesinato de Eugenio Garza Sada, México, Grijalbo, 2006, p.p. 15 y 48

${ }^{50}$ Mario Ramírez Salas (integrante del tercer comando) en Zamora, Michoacán el 29 y 30 de julio del 2002, Entrevista. Mario Ramírez Salas se hospedo durante varios días, en la casa del jesuita y profesor del ITESM, Javier de Obeso, durante la redada y persecución que sufrieron los miembros guerrilleros de los Procesos. Logró salir de Monterrey, regresar a la ciudad de México y posteriormente en agosto de ese año, trasladarse con otros guerrilleros a la sierra de Guerrero, donde tenían la misión dada por Salas Obregón, de buscar una alianza con el Partido de los Pobres de Lucio Cabañas

${ }^{51}$ Véase HEP, El Porvenir y HEN, El Norte, Monterrey, de 15 al 23 de enero de 1972, p.p. 1 y 2

${ }^{52}$ Gustavo Hirales nos dejó cuatro textos escritos sobre los acontecimientos de esta época. El de más impacto es Gustavo Hirales HIRALES, Gustavo, 1996, La guerra de los justos, México, Editorial Cal y Arena, 1996

${ }^{53}$ Declaración preparatoria de los acusados ante el Juez Cuarto Penal, Marco Antonio Leija Moreno, Monterrey, 21 de enero de 1972, en O. Flores, "Del Movimiento universitario", p.p. 480 y 481 
incautaron armas de fuego. En una de estas aprehensiones, se dio un enfrentamiento armado de consideración entre la guerrilla descubierta y la policía. El enfrentamiento que conmocionó a la opinión pública se dio en el Edificio número 7 de los "Condominios Constitución".

La Universidad Autónoma de Nuevo León, no estuvo al margen de los acontecimientos, en enero de 1972 y antes de la balacera en los Condominios Constitución, el director de la Facultad de Ciencias Químicas descubrió en las instalaciones de la mencionada institución, un arsenal de armas, municiones y bombas molotov empaquetadas en una de sus bodegas. El aviso oportuno a las autoridades estatales desactivó un probable acontecimiento mayor.54

El rector Héctor Ulises, manifestó a la opinión pública el sentir de las autoridades universitarias por los últimos acontecimientos sucedidos en la ciudad, donde numerosos universitarios habían resultaron involucrados. 55 Sin embargo, nuevos actos de violencia y muerte estaban por venir. Dos acontecimientos guerrilleros más, sucedido uno a fines de 1972 y otro en septiembre de 1973, conmocionaron a la ciudad de Monterrey y a la nación.

El primero de ellos, fue el acaecido el 8 de noviembre de 1972, día que se llevó a cabo el secuestro aéreo más espectacular realizado en el país, así como uno de los primeros en su tipo en el mundo. $5^{6}$ En efecto, el grupo guerrillero Liga de Comunistas Armados, liderados por Germán Segovia, alias Raúl, secuestraron el vuelo 705 de Mexicana de Aviación en el aeropuerto Mariano Escobedo con destino a la ciudad de México. $57 \mathrm{El}$ secuestro hecho de una forma espectacular, obligó dirigir el avión hacia

\footnotetext{
${ }^{54}$ Véase la entrevista con Pablo Morales Pinal (director de la Facultad de Ciencias Químicas de diciembre de 1971 a 1973), "Un director polémico", en Proyecto UANL, revista mensual, año 1, número 3, San Nicolás de los Garza, Universidad Autónoma de Nuevo León (UANL), 1995, p. 12

${ }_{55}^{5}$ HEN, El Norte, "Rectoría a la Comunidad Universitaria", Monterrey, 20 de enero de 1972, p. 7-A

${ }^{56} \mathrm{El} 2$ de agosto de 1970, se presentó el primer secuestro aéreo de la historia, cuando un Boeing 747 fue desviado mientras volaba de Nueva York a Puerto Rico. O. Flores, (2008), "Del movimiento universitario", p.p. 484-485.

57 Véase HEP, El Porvenir 9 de noviembre 1972, p. 1 y HEN, El Norte, 9 de noviembre de 1972, página 1. Ambos diarios dieron esta información en la página principal del periódico. Entre las personalidades políticas y empresariales de la localidad que se encontraban en ese avión, estaban, Jorge Fernández Ruiloba y Eugenio Garza Botello del Centro Patronal; Dionisio Garza Sada y Francisco González quienes asistirían a la Asamblea de COPARMEX, a llevarse a cabo en la ciudad de Cuer-
} 
la isla de Cuba, y posteriormente, el gobierno de éste país, facilitó el regreso de todos sus ocupantes a Monterrey. 58

El segundo acontecimiento, está relacionado con la primera acción guerrillera a escala nacional que implementó la recién fundada Liga Comunista 23 de Septiembre. Considerando esta organización tener suficientes fuerzas para realizar acciones electrizantes y de gran impacto en la comunidad empresarial, un comando de la Liga intentó secuestrar a uno de los empresarios más prominentes del país con residencia en Monterrey. Si bien, un año atrás, el grupo de los Procesos ya tenía contemplado el secuestro de seis posibles empresarios a fin de pedir un cuantioso rescate (10 a 20 millones de pesos), entre los que se encontraban los nombres de Camilo Sada, Manuel Barragán, Carlos Prieto, Eugenio Garza Lagüera, Alejandro Garza Lagüera y Eugenio Garza Sada, el Comité Nacional de la Liga optó por éste último.59 El 17 de septiembre de 1973, se llevó a cabo la acción bajo el comando dirigido por Edmundo Medina Flores. Los otros integrantes fueron Juan Corral, Hilario Juárez García, Javier Rodríguez Torres y Hernando Martínez. Interceptado el vehículo del empresario y tras una balacera, resultó fallido el intento de secuestro y muertos Eugenio Garza Sada, su chofer (Bernardo Chapa) y un guardaespaldas (Modesto Torres), por su parte, la Liga perdió a dos de sus miembros (Javier Rodríguez Torres y Hernando Martínez), encontrados muertos dentro de un automóvil modelo "Falcon", a las 11 horas del mismo día. ${ }^{60}$ El primer informe sobre la muerte de Eugenio Garza Sada es elaborado horas después de los hechos por el propio director de la Dirección Federal de Seguridad (DFS), Luis de la Barrera Moreno, a sus superiores, el secretario Moya Palencia y al presidente Luis Echeverría. 61

Menos de un mes después, otro comando de la Liga secuestró en Guadalajara al empresario Fernando Aranguren y al cónsul honorario británico Duncan Williams. Las negociaciones para obtener el rescate de ambos fracasaron, por lo que el

\footnotetext{
navaca, Morelos; Graciano Bortoni; Gonzalo Estrada; Wesley H. Parsons, del consulado de los Estados Unidos de América en Monterrey; Oscar y Rooselvet Guerra; Jaime Llaguno; Pablo Madero; Jesús y Roberto Zambrano, Rogelio Elizondo y el profesor e investigador de la UNAM, Santiago Genovés, entre otros.

${ }^{58}$ Véase HEP, El Porvenir 10 de noviembre 1972, p. 1 y HEN, El Norte, 10 de noviembre de 1972, p. 1

${ }^{59}$ F. Menéndez, Nadie supo nada, 2006, p.p. 15

${ }^{60}$ F. Menéndez, Nadie supo nada, 2006, p.p. 46 y 47

${ }^{61}$ Luis Barrera Moreno, Dirección Federal de Seguridad, "Informe de la muerte de Eugenio Garza Sada", expediente 11-219-972, legajo 2, hojas 46-47, Archivo General de la Nación (AGN), México.
} 
comando que retenía a ambos secuestrados, tomó la decisión sin consultar al dirigente nacional Salas Obregón, de dejar en libertad al cónsul Williams, pero ejecutar a Aranguren. Esta decisión enfadó a la dirigencia nacional, la cual hubiera preferido realizar ambas ejecuciones, por lo que decidió no pasar por alto esta falta y dar un castigo ejemplar. El líder del comando llamado Pedro Orozco, murió acribillado días después. La respuesta del Estado fue igual de brutal. Miembros de la Dirección Federal de Seguridad, que comandaba Miguel Nassar Haro, localizaron y aprehendieron a dos miembros de la Liga que participaron en los secuestros: a Sebas (Ignacio Olivares) y a Roberto (Salvador Corral).

A raíz de estos acontecimientos, podemos asegurar, que la trágicamente famosa guerra sucia en nuestro país, había comenzado.

No hay duda, que el programa político que el presidente Luis Echeverría llevó a cabo tuvo sus timbres de éxito. Tal vez el más importante, fue el diluir los efectos negativos del 68 en múltiples sectores. Sin embargo, fue ineficaz ante la izquierda radical de origen universitario que para 1972-1973 había recurrido a la violencia.

Este sector de oposición se convirtió rápidamente en una guerrilla urbana, producto de la actividad de estudiantes y profesores disidentes de las diferentes corrientes de izquierda y de organizaciones cristianas, que después de los trágicos sucesos en México de 1968, pasaron de las acciones críticas abiertas y legales a las vías de hecho y clandestinas. Aunque en ocasiones el Partido Comunista Mexicano se adjudicó un cierto afán en ver en esta lucha armada un resultado de su labor62, su control sobre ella fue meramente demagógico. En otras palabras, terminaron adjudicándose hechos que nunca previeron ni controlaron dentro de la heterogénea gama de la izquierda mexicana.

Por otra parte, la represión oficial desarticuló el movimiento estudiantil en la capital del país y en otras ciudades, como fue el caso de Monterrey. Pero a la vez propició la creación de una nueva cultura política radical de izquierda que optó por la lucha armada. La guerrilla en México no era nueva. Durante la década de los años sesenta la guerrilla rural en Guerrero, encabezada por Lucio Cabañas y Genaro Vázquez, fueron un ejemplo de ello. Pero la guerrilla urbana de los setenta era diferente, la organización más importante fue la Liga Comunista 23 de Septiembre. La Liga

\footnotetext{
${ }^{62}$ Valentín Campa, Mi testimonio. Memorias de un comunista mexicano, México, Ediciones de Cultura Popular, 1978, p.p. 67-73.
} 
tuvo una actividad significativa en la entidad federativa de Nuevo León y en igual proporción en otros estados de la república. Su rápida descomposición se dio a partir de 1973, a raíz de la muerte del empresario y capitán de industria Eugenio Garza Sada y del asesinato del industrial Fernando Aranguren en Guadalajara.

\section{Comentario Final}

Podemos concluir después de este sucinto recorrido histórico, que una de las significantes centrales del movimiento contra los textos gratuitos en Monterrey en 1962, y posteriormente el movimiento contestatario popular de 1968 en la ciudad de México y 1969-1973 en Monterrey, fue su pretensión de convertirse en pionero de la lucha por la conquista del derecho de los distintos grupos y clases sociales a organizarse sin la tutela estatal. En el caso de Monterrey, esta pretensión tuvo una fuerza importante al inicio del conflicto, sin embargo, rápidamente cayó presa de la propia tutela estatal (en este caso de las prebendas dadas por el gobierno federal en $1963 \mathrm{y}$ posteriormente entre 1971 y 1972), en su intento de enfrentar al proyecto conservador de Universidad pública, que planteó el gobierno de Elizondo y la comunidad industrial de esta ciudad.

Por su parte, el proyecto conservador de la elite política-económica de Monterrey quedo restringido a Nuevo León durante varios años. Sin embargo, a partir de la década de los ochenta y particularmente a raíz del triunfo del Partido Acción Nacional en las elecciones para presidente de la república en el año 2000, el proyecto educativo, político y económico original de los Grupos empresariales de Monterrey (con algunos ajustes, claro está) fue consolidado a nivel nacional. A partir de la segunda mitad del año de 2000, la capital de la república y sede de los poderes de la Unión, vio la llegada de numerosos asesores egresados de las Instituciones de Educación Superior privadas de Monterrey y de la UANL, destinados al sector económico $\mathrm{y}$ al sector educativo del gobierno federal Un ejemplo de ello es el caso de Reyes Tamez, rector de la UANL, quién fue invitado al cargo de Secretario de Educación para el sexenio 2000-2006. En el caso del gobernador Fernando Canales Clarión (un próspero empresario que ganó las elecciones estatales en 1997), renunció en enero de 2003, como encargado del poder ejecutivo de Nuevo León, para ocupar la Secretaría de Economía en el gabinete nacional. Ante su renuncia, el Congreso estatal designó como gobernador interino a Fernando Elizondo Barragán, miembro entre 
otros organismos del PAN, de numerosos consejos ejecutivos de destacadas empresas e Instituciones de Educación Superior Privadas, e hijo por supuesto, del exgobernador Eduardo A. Elizondo.

Por otra parte, en octubre del año 2001, el gobierno del presidente Vicente Fox creó la Fiscalía Especial para Movimientos Sociales y Políticos del Pasado, a fin de esclarecer las desapariciones de la guerra sucia. Esta Fiscalía -a cargo del fiscal especial Ignacio Carrillo Prieto-, trabajó sobre la primera averiguación previa que abrió la dependencia y que consistía en la investigación de 531 presuntos desaparecidos. Para el 1 de septiembre de 2002, la dependencia había recibido un número creciente de quejas y agravios, sembrados en los días del autoritarismo del antiguo régimen priísta. Por lo que habrá que agregar las pesquisas sobre las matanzas de 1968 y 1971; el asesinato de 658 militantes del PRD y el número indeterminado de profesores miembros del llamado magisterio democrático. Sabemos que la muerte de Digna Ochoa en el año de 2001, catapultó la decisión del nuevo gobierno de esclarecer estos hechos y entregar parte de los Archivos de la Secretaría de la Defensa del Centro de Investigación y Seguridad Nacional (Cisen) y de la Secretaría de Gobernación al Archivo General de la Nación (AGN). En total, entre el 19 y 21 de febrero del 2002, fueron entregadas al AGN 4 mil cajas del Cisen. De estas cajas, se calcula que son 80 millones de tarjetas con documentación de 4 millones de actores políticos, además de 26 mil videos y 250 mil fotografías.

\section{Bibliografía:}

\section{Fuentes Primarias}

Archivo General de la Nación (AGN), México, Expediente 11-219-972, legajo 2, hojas 46-47 de la desaparecida Dirección Federal de Seguridad (DFS), ahora en el AGN.

Hemeroteca del Periódico El Norte (HEN), Monterrey, México.

Hemeroteca del Periódico El Porvenir (HDP), Monterrey, México 
Entrevistas

Entrevista de Oscar Flores con Sergio Aguayo el 31 de julio de 2002, en Zamora, Michoacán

Entrevistas de Oscar Flores con Mario Ramírez Salas el 29 y 30 de julio del 2002, en Zamora, Michoacán

\section{Fuentes Primarias Impresas}

Dirección General de Estadística, Censos Generales de Población, 1960, 1970, 1980 y 1990, INEGI, México, D.F.

\section{Publicaciones periódicas}

Hemeroteca del Periódico El Norte, Monterrey, marzo-diciembre de 1946, pág. 1 y 2 A

Hemeroteca del Periódico El Norte, Monterrey, 2 y 3 de febrero de 1962, p.1;

Hemeroteca del Periódico El Norte, Monterrey, 19 de enero de 1968, 1-B;

Hemeroteca del Periódico El Norte, Monterrey, 24 de octubre de 1969, 1 y 4-B;

Hemeroteca del Periódico El Norte, Monterrey, 5 de noviembre de 1969, 11-B;

"El Proyecto de Reforma a los Artículos 16 y 24 de la Ley Orgánica", Hemeroteca del Periódico El Norte, Monterrey, 10 de noviembre de 1969, p. 1-B;

Hemeroteca del Periódico El Norte, Monterrey, 11 de noviembre de 1969 p.p. 1 y 7 B, 9 y 11 ;

Hemeroteca del Periódico El Norte, Monterrey, 19 de noviembre de 1969, 1-B, 21 de mayo de 1970;

"Confiesa dirigir doble asalto. Ruiz Díaz hunde a sus cómplices”, Hemeroteca del Periódico El Norte, Monterrey, 18 de enero de 1972, p. 8-A;

Hemeroteca del Periódico El Norte, Monterrey 19 al 23 de enero y 9, 10 y 30 de noviembre de 1972, p. 2-B;

"Rectoría a la Comunidad Universitaria”, Hemeroteca del Periódico El Norte, Monterrey, 20 de enero de 1972, p. 7-A 
Beltrán del Río, Pascal, "Desaparecidos: una impunidad que peina canas”, Hemeroteca del Periódico El Norte, Monterrey, 1 de septiembre de 2002, p. 12A

Hemeroteca periódico El Porvenir, Sección Local p.1 11 de noviembre de 1969 y 9 y 15 al 23 de enero y 9 y 10 de noviembre de 1972

Hemeroteca del Periódico El Norte, 1B, Monterrey, 11 de noviembre de 1969 y 9 y 15 al 23 de enero y 9 y 10 de noviembre de 1972

Periódico Oficial del Estado de Nuevo León, Gobierno del Estado de Nuevo León, Monterrey, 11 de noviembre de 1969, p. 9-11

Periódico Oficial del Estado de Nuevo León, Gobierno del Estado de Nuevo León, Monterrey, 26 de noviembre de 1968, p.p. 6-8.

"Un director polémico", Entrevista con Pablo Morales Pinal (director de la Facultad de Ciencias Químicas de diciembre de 1971 a 1973), en Proyecto UANL revista mensual, año 1, número 3, 1995, pp. 12-18

\section{Fuentes Secundarias}

Aguayo, Sergio, La Charola. Una historia de los servicios de inteligencia en México, México, Grijalbo, 2001.

Aguilar Camín, Héctor, Subversiones silenciosas: ensayos de historia y política de México, México, Aguilar Nuevo Siglo, 1994.

Aguilar Camín, Héctor, La guerra de Galio, México, Cal y Arena , 1992

Aguilar Camín, Héctor, Después del milagro, México, Cal y Arena, 1989

Ávila, Ana Cristina y Virgilio Muñoz, Creación de la Comisión Nacional de Libros de Texto Gratuitos: La perspectiva escolar, 1958-1964, México, Noriega Editores, 1999, pp. 87-112

Armendáriz Ponce, Minerva, Morir de sed junto a la fuente, México, Edición de la autora (Testimonio), 2001

Campa, Valentín, Mi testimonio. Memorias de un comunista mexicano, México, Ediciones de Cultura Popular, p.p. 1978.

Esteve Díaz, Hugo, Las armas de la utopía. La tercera ola de los movimientos guerrilleros en México, México, Instituto de Proposiciones Estratégicas, 1996

Fernández Menéndez, Jorge, Nadie supo nada. La verdadera historia del asesinato de Eugenio Garza Sada, México, Grijalbo, 2006

Flores, Oscar, La Autonomía Universitaria, 1968-1971, México, UANL, 2011. 
Flores, Oscar, "Del movimiento universitario a la guerrilla. El caso de Monterrey (1968-1973)”, en Verónica Oikión y Marta Eugenia García, Movimientos armados en México, Siglo XX, Zamora, Michoacán, El Colegio de MichoacánCIESAS, Volumen II, 2008, p.p. 461-494.

Flores, Oscar, Historiadores del México Siglo XX, Selección, presentación y notas de, México, Editorial Trillas, Colección Linterna Mágica, número 33, 2003, p.p. 112-125.

Flores, Oscar, Universidad de Monterrey. Historia y Desafios (1969-2004), Editorial Trillas, México, 2004.

García, Susana y Vanella, Liliana, Política educativa y valores nacionales, México, Nueva Imagen, 1979.

Guevara Niebla, Gilberto, La democracia en la calle, México, Siglo XXI Editores, 1986.

Guevara Niebla, Gilberto, "El 68 y la Universidad", en Revista de la Universidad de México, 4 - 5, diciembre de 1978-enero, 1979, México, p.p. 1-4.

González de Alba, Luis, Los días y los años, México, Era-SEP, 1971

Ramírez, Ramón, El movimiento estudiantil de México, julio-diciembre de 1968, 2 vols. México, Era, 1969

González Pedrero, Enrique, Los libros de texto gratuito, México, SEP, 1982

González Rubio, Javier (coordinador), México, 30 años de movimiento, México, Universidad Iberoamericana, 1998

Graves Laine, Cecilia, "Política educativa y libros de texto gratuitos. Una polémica en torno al control por la educación”, en Revista Mexicana de Investigación Educativa, mayo-agosto, volumen 6, número 12, Consejo Mexicano de Investigación Educativa, México, 2001, p.p. 205-221.

Hirales, Gustavo, La guerra de los justos, México, Editorial Cal y Arena, 1996.

Latapí, Pablo, Política educativa y valores nacionales, Editorial Nueva Imagen, México, 1979.

Ortega, Olivares, Octubre dos: historias del movimiento estudiantil, México, UAMUnidad Xochimilco, 1998.

Raby, David L. "La "Educación socialista” en México", en Cuadernos Políticos, número 29, México, D. F., Editorial Era, julio-septiembre de 1981, pp. 75-82.

Ramírez Salas, Mario, "La relación de la Liga Comunista 23 de septiembre y el Partido de los Pobres en el Estado de Guerrero en la década de los 70's”, trabajo 
presentado en el Foro de Discusión Académica La guerrilla en las regiones de México, siglo XX, llevado a cabo en El Colegio de Michoacán, Zamora, Michoacán, México, del 29 al 31 de julio de 2002, p. 1-28.

Ramírez, Ramón, El movimiento estudiantil de México, julio-diciembre de 1968, 2 vols. México, Era, 1969

Sierra, José Luis, "Fuerzas armadas y contrainsurgencia (1965-1982), Foro de Discusión Académica La guerrilla en las regiones de México, siglo XX, llevado a cabo en El Colegio de Michoacán, Zamora, Michoacán, México, del 29 al 31 de julio de 2002, p.p. 1-32.

Zermeño, Sergio, México: una democracia utópica. El movimiento estudiantil del 68, México, Siglo XXI, 1978. 